Revue d'histoire de l'enfance « irrégulière »

Le Temps de l'histoire

6 | 2004

Les sciences du psychisme et l'enfance « irrégulière »

Nathalie Bélanger De la psychologie scolaire à la politique de l'enfance inadaptée

Préface d'Eric Plaisance Paris, éditions du CTNERHI, 2002, 148 p.

Elisabeth Callu

(2) OpenEdition

Journals

Édition électronique

URL : http://journals.openedition.org/rhei/124

DOI : $10.4000 /$ rhei. 124

ISBN : 978-2-7535-1644-1

ISSN : 1777-540X

Éditeur

Presses universitaires de Rennes

Édition imprimée

Date de publication : 15 novembre 2004

Pagination : 293-299

ISSN : 1287-2431

Référence électronique

Elisabeth Callu, « Nathalie Bélanger De la psychologie scolaire à la politique de l'enfance inadaptée »,

Revue d'histoire de l'enfance « irrégulière » [En ligne], 6 | 2004, mis en ligne le 15 novembre 2004,

consulté le 04 décembre 2020. URL : http://journals.openedition.org/rhei/124 ; DOI : https://doi.org/

10.4000/rhei.124 


\author{
Nathalie Bélanger \\ De la psychologie scolaire à la politique de l'enfance inadaptée \\ Préface de Éric Plaisance \\ Paris, éditions du CTNERHI, 2002, 148 p.
}

Avec De la psychologie scolaire à la politique de l'enfance inadaptée, Nathalie Bélanger nous découvre un épisode de l'histoire peu étudiée des politiques et des mesures en faveur de l'enfance inadaptée, dite plus souvent aujourd'hui handicapée. Plus exactement, il nous en fait parcourir un détour, puisque son titre ne suggère pas une démarche chronologique mais plutôt un itinéraire qui, partant de l'observation de la genèse et du développement de la psychologie scolaire en France, nous ramène constamment à la naissance du secteur de l'éducation spécialisée. La thèse centrale de l'ouvrage est, en effet, que le corps professionnel et les pratiques des psychologues scolaires n'ont pu se construire et perdurer à partir des années soixante qu'en prenant appui sur la construction et la pérennisation concomitante de ce secteur de l'éducation spécialisée. Cette dernière y est principalement entendue dans son acception scolaire (classes de perfectionnement, enseignement spécial...), même si l'auteure fait à plusieurs reprises référence au développement parallèle de l'éducation spécialisée dans le secteur médicosocial privé, grâce au soutien du ministère de la Santé... et aux subsides de la toute jeune Sécurité sociale.

Le travail de Nathalie Bélanger, aujourd'hui professeur à l'Institut d'études pédagogiques de l'Ontario, est principalement fondé sur l'analyse de revues professionnelles, l'exploitation d'archives inédites - notamment celles du collectif Histoire de la psychologie scolaire - et sur des entretiens avec des " personnalités ayant joué un rôle dans l'histoire de la psychologie scolaire ». Deux enquêtes par questionnaires réalisées auprès de deux "générations" de professionnels (1945-1954 et 1960-1970) viennent compléter les archives écrites et orales.

La chercheuse s'intéresse d'abord aux sources théoriques plus anciennes du projet d'un service de psychologie scolaire mis en forme en 1945 dans le cadre du Plan Langevin-Wallon. Au-delà de la nécessité de bien connaître

Élisabeth Callu / p. 293 à 299 
l'enfant que l'on éduque, affirmée avec force par les grands pédagogues depuis la Renaissance, s'est développée peu à peu, à la fin du XIXème siècle, l'idée d'une rationalisation de l'éducation : mieux connaitre le développement de l'enfant, en général, et identifier les aptitudes de chacun, en particulier, permettra d'adapter l'enseignement en vue d'une meilleure efficacité. La psychologie de l'éducation devient une "science d'utilité sociale ", écrivent en 1908 Alfred Binet et Théodore Simon, et cette science stratégique doit être reliée aux politiques éducatives. Cette conviction que la science peut permettre de rationaliser l'activité humaine et améliorer le « rendement national » anime les auteurs et les défenseurs du Plan, notamment lorsque celui-ci préconise la mise en place de psychologues dans les écoles. En effet, l'institution scolaire doit faire face aux problèmes qu'elle rencontre " du fait du progrès et de la spécialisation des connaissances, de la complexité croissante des structures sociales et des possibilités plus diverses auxquelles elle prépare ». Dans une perspective plus politique, il lui faut donc "connaître objectivement les raisons "intellectuelles", caractérielles ou sociales des comportements et des "infériorités" des enfants socialement défavorisés ", afin de mieux adapter la pédagogie. Autrement dit, il faut mesurer les conséquences positives et négatives des différentes méthodes et les adapter - tout comme les programmes - au développement de l'enfant, pour combattre "l'iniquité d'un système scolaire fondé sur la ségrégation sociale " en abattant " les hautes et solides barrières qui, sur les voies de la culture, se [dressent] devant la jeunesse ouvrière et paysanne». En bref, il s'agit « $\left[d^{\prime}\right]$ aider à la meilleure adaptation de l'élève à l'école, à la meilleure appropriation de la vie scolaire aux intérêts de l'enfant». Pour mettre en œuvre une telle politique, il faudra que les psychologues scolaires soient eux-mêmes des pédagogues, recrutés parmi les membres expérimentés du corps enseignant, par ailleurs formés à la psychologie.

Les tâches réellement effectuées par les psychologues scolaires de la première génération (1945/1954) sont mal connues. Les rares textes existant sur le sujet sont très généraux. Ils permettent toutefois à Nathalie Bélanger de nous préciser les objectifs assignés à ces pionniers de la profession : " établir pour tous les élèves nouveaux un dossier psychopédagogique », " satisfaire à toutes les demandes d'examen présentées par le corps enseignant » 
et « amorcer des recherches sur la psychopédagogie des matières d'enseignement ". La connaissance des enfants est donc recherchée dans une perspective "scientifique" d'amélioration de l'enseignement, non dans celle d'un dépistage des inadaptés : "les cas individuels d'inadaptation scolaire ", les retards scolaires - notamment en français et mathématiques, qui suscitent un nombre important d'articles dans les revues spécialisées de l'époque sont étudiés pour envisager, " par une étroite collaboration avec les pédagogues, [...] les solutions les plus appropriées ». Il faut pour cela des outils, et l'un des témoins interrogés par la sociologue précise que, les premières années, lui-même et ses collègues ont consacré beaucoup de temps à l'étude, l'adaptation et l'étalonnage de tests, souvent américains, la psychologie d'Outre-Atlantique étant alors prépondérante dans la construction du modèle français de psychologie scolaire.

L'expérimentation de ce premier modèle, fondé sur la volonté d'améliorer les pratiques pédagogiques en s'appuyant sur la connaissance psychologique de l'enfant et de son développement, a été brève. Elle fut interrompue - à tout le moins mise en veilleuse - assez brutalement, par un texte ministériel de 1954. Selon les acteurs concernés, ce texte résultait de l'effet conjugué du baby-boom - nécessité/prétexte de réintégrer à leurs postes d'enseignant tous les personnels affectés à d'autres tâches - et de la guerre froide - mise à l'écart du réseau des psychologues scolaires recrutés voire cooptés par Henri Wallon et René Zazzo, eux-mêmes membres du PCF, et réputés diffuser les idées communistes dans l'institution scolaire. Sans nier cette double contingence, Nathalie Bélanger reprend également à son compte l'hypothèse de Guy Avanzini sur les sources plus fondamentales de cette remise en cause : les ambiguités intrinsèques au Plan LangevinWallon. Celui-ci se serait de toute façon avéré inapplicable car traversé par de nombreuses questions non résolues - par exemple : quels résultats concrets vise-t-on lorsqu'on veut promouvoir à l'école « la justice, l'égalité et la démocratie " ? -, voire quelques contradictions : comment concilier entre autres - l'attention aux " aptitudes individuelles ", préconisée par le courant influent de l'éducation nouvelle, et la volonté affirmée de rationaliser l'enseignement et l'orientation professionnelle en fonction de la situation socio-économique du pays ? Paradoxalement, ce sera pendant les 
années suivantes - où la psychologie scolaire est en quelque sorte mise en latence - que le "modèle français" inspirera les recommandations de l'UNESCO en la matière et sera repris dans plusieurs pays.

Malgré le déclin engendré par la décision de 1954, ce modèle n'a pas vraiment cessé d'exister : certes, une cinquantaine de psychologues ont dû regagner leur poste d'instituteur et certains sont partis vers les centres psycho-pédagogiques (première version des futurs CMPP) créés par Henri Wallon, mais d'autres ont pu conserver leur poste ou, n'étant pas titulaires, ont vu leurs contrats renouvelés d'année en année. La psychologie scolaire va donc pouvoir réémerger à la fin des années cinquante et se développer au cours de la décennie qui suivra, mais cet essor s'appuiera principalement sur la préoccupation grandissante du ministère de l'Éducation nationale et du Gouvernement concernant l'enfance inadaptée. En 1958, une circulaire mentionne la présence requise du psychologue scolaire, "lorsqu'il en existe un dans le département ", au sein de la commission médico-pédagogique chargée de déterminer " quels élèves ne peuvent être admis ou maintenus dans les classes primaires publiques et, parmi eux, ceux qui peuvent être reçus dans une classe ou une école spéciale ». C’est désormais l'éducation spéciale qui semble permettre à l'identité professionnelle des psychologues scolaires de se cristalliser progressivement autour de l'étude et de l'orientation des "enfants à problèmes". Après tout, n'est-ce pas un retour aux sources de la psychologie scolaire, vues sous une autre facette ? Autant qu'à la promotion d'une psychologie individuelle au service de l'éducation et d'une instruction adaptée aux aptitudes, Alfred Binet n'avait-il pas, nous dit l'auteure, œuvré à la définition de la normalité et de l'adaptation - i.e. de l'anormalité et de l'inadaptation -, " notions fondatrices de la psychologie scolaire » dans sa version actuelle?

En 1960, une note de service de l'Éducation nationale reprécise pourtant le rôle et les activités du psychologue scolaire dans une perspective plus conforme aux orientations initiales d'Henri Wallon : suivre le développement de chaque enfant pour faciliter, le temps venu, son orientation. Elle mentionne néanmoins le rôle spécifique de ce corps professionnel dans le dépistage des enfants inadaptés, le fonctionnement de la commission médico-pédagogique et celui des classes spéciales. En fait, ce que laisse entendre 
Nathalie Bélanger - sans toutefois le formuler très explicitement -, c'est que la construction du noyau dur de l'identité professionnelle des psychologues scolaires s'est faite selon une double polarisation, positive et négative : d'une part, comme on l'a vu, en réponse à l'attraction exercée par les attentes institutionnelles fortes d'un secteur de l'éducation spéciale en voie de constitution ; d'autre part, "en creux", par abandon d'autres contenus professionnels possibles, mais moins clairement repérés et formalisés (suivi individuel de tous les élèves, participation à l'orientation, contribution à une meilleure adaptation de la pédagogie), sous la triple pression :

- de la demande d'aide des enseignants, dépassés par certains élèves "dyslexiques ", "à problèmes ", voire " inadaptés ", dans un contexte de classes souvent surchargées ;

- de la résistance passive ou active de ces collègues à une intervention des psychologues en matière de pédagogie ;

- de luttes corporatistes, tantôt ouvertes, tantôt larvées, entre le corps des psychologues et celui des conseillers d'orientation.

Cela se fera, semble-t-il, au corps défendant des psychologues scolaires eux-mêmes. Les pionniers, comme les nouveaux venus, ne semblent pas avoir trouvé dans les expérimentations de la première période de quoi progresser vers une définition de leurs fonctions et tâches plus conforme au projet originel - qui, au moins pour les plus anciens, semble prendre valeur de mythe - ni dégagé la marge de manœuvre pour expérimenter à leur tour. Pour l'auteure, la tentation aura été trop forte de conforter leur place là où ils étaient le plus attendus, même si cette adaptation a pu prendre la tonalité d'une trahison des idéaux fondateurs.

Le lecteur l'aura compris, Nathalie Blanchard récuse d'emblée l'hypothèse - largement diffusée aujourd'hui, nous dit-elle, parmi les psychologues scolaires - d'un développement linéaire du corps professionnel répondant progressivement à un besoin naissant de l'institution scolaire, à l'issue de la seconde guerre mondiale. Sa perspective d'analyse est résolument "constructiviste" et s'intéresse essentiellement aux interactions entre les différents facteurs en jeu dans l'élaboration croisée de la réponse : la psychologie scolaire, et du problème : le dépistage et l'orientation des enfants inadaptés à l'école. Elle énumère dans sa conclusion les facteurs suivants : 
le rôle des groupes d'acteurs et des réseaux, et notamment les effets liés à l'émergence d'un nouveau corps professionnel ; le contexte politique, voire géopolitique ; les caractéristiques personnelles et biographiques des acteurs, évoquées passim dans l'ouvrage ; les effets structurels et institutionnels de la mise en œuvre du projet lui-même.

Si la présentation et la démonstration de la thèse ne manquent pas d'intérêt, offrant un point de vue relativement inédit sur l'histoire des politiques de l'inadaptation, appuyé sur des documents peu connus ou inconnus pour la plupart, le lecteur se trouve parfois gêné par l'absence de choix entre une présentation des données de type historiographique et de type problématique. La présentation thématique à l'appui de la thèse est affaiblie par l'enchevêtrement avec d'autres éléments concomitants ; l'articulation chronologique des discours et des faits (principalement des décisions administratives, la réalité des tâches professionnelles, par exemple, n'apparaissant qu'à travers une des enquêtes présentées) est difficilement lisible du fait de multiples allers et retours dans le temps. C'est dommage car une analyse des différentes conceptions de la psychologie scolaire moins éparpillée entre les différentes périodes et les différentes sources - elles-mêmes hétérogènes - aurait peut-être permis au béotien en la matière - ici un peu perdu - de mieux comprendre, par exemple, la finesse des distinctions établies par l'auteure entre des positions - apparemment proches en première lecture - et pourtant analysées par l'auteure comme révélatrices de points de vue nettement divergents. En quoi, par exemple, une psychologie scolaire qui vise une prévention de l'inadaptation interne à l'école, ayant pour objet d'éviter une orientation vers le secteur de l'éducation spécialisée, constitue-t-elle une trahison du projet "wallonnien" d'adaptation de l'élève à l'école et de la vie scolaire à l'enfant?

Enfin, du fait des sources utilisées, le propos de l'auteure se trouve très centré sur les questions posées autour de l'inadaptation au sein de l'Éducation nationale et sur la manière dont elles ont été résolues par l'institution. Néanmoins, le grand intérêt de ce travail est peut-être, à partir des repères historiques et des éclairages apportés sur ce point, de permettre à d'autres de proposer de nouvelles hypothèses sur les interactions avec le contexte externe à l'institution scolaire, et notamment celui - évoqué - du monde 
de la Santé et des Affaires sociales : comment interpréter les différentes coïncidences temporelles dans la construction des dispositifs scolaires et médico-sociaux de l'éducation spéciale ? Y a-t-il des connexions entre les différents réseaux d'acteurs, qui apparaissent ici disjoints, à travers quels personnages clés ou réseaux intermédiaires ? La concurrence secteur scolaire public/médico-social privé, rapidement évoquée, a-t-elle donné matière à débats publics ou au sein de groupes plus fermés ?...

Je terminerai sur une question : l'ouvrage présenté ici nous propose un certain nombre de sources et de grilles d'analyse pour comprendre et définir différentes conceptions, d'une part, de la collaboration entre psychologues et pédagogues, d'autre part, de l'information du champ de la pratique éducative par les connaissances relevant du champ disciplinaire de la psychologie ; ne pourraient-elles s'avérer profitables pour les lecteurs qui s'intéressent à ces questions du point de vue de l'éducation spécialisée dans son versant social ou médico-social ?

\section{Élisabeth Callu}

